K. Hayasida and Y. Ikeda

Nagoya Math. J.

Vol. 92 (1983), 51-67

\title{
ON POSITIVE VECTOR SUPERFUNCTIONS OF ELLIPTIC SYSTEMS
}

\section{KAZUYA HAYASIDA AND YOSHIAKI IKEDA}

1. Let $\Omega$ be a bounded domain in $R^{n}$ with coordinates $x=\left(x_{1}, \cdots, x_{n}\right)$ such that $\Omega$ contains the origin. We consider the elliptic operators

$$
L_{k}=\sum_{i, j=1}^{n} a_{i j}^{(k)}(x) \partial_{x_{i}} \partial_{x_{j}}, \quad k=1,2, \cdots, m,
$$

where $a_{i j}^{(k)}$ are real valued and in $C^{2}(\Omega)$. And we assume that there are positive constants $c_{0}, c_{1}$ with $n c_{1}-2 c_{0}<(n-1) \cdot c_{0}$ such that for any $x \in$ $\Omega$ and any $\xi \in R^{n}$

$$
\sum_{i, j=1}^{n} a_{i j}^{(k)}(x) \xi_{i} \xi_{j} \geqq c_{0}|\xi|^{2}, \quad 0<a_{i i}^{(k)}(x) \leqq c_{1} .
$$

Obviously $c_{0} \leqq c_{1}$.

In this article we consider the solutions $\left\{u_{k}\right\}$ of the elliptic differential inequalities in $\Omega$

$$
L_{k} u_{k} \leqq \sum_{\ell=1}^{m} \sum_{i=1}^{n} b_{i \ell}^{(k)}(x) \partial_{x_{i}} u_{\ell}+\sum_{\ell=1}^{m} c_{\ell}^{(k)}(x) u_{\ell}, \quad k=1,2, \cdots, m .
$$

Here all coefficients are real valued and

$$
b_{i \ell}^{(k)} \in C^{1}(\Omega), \quad c_{\ell}^{(k)} \in L_{\mathrm{loc}}^{\infty}(\Omega) .
$$

The assumption on $c_{\ell}^{(k)}$ will be weakened later (see Remark 1 in the final section).

Let $F$ be a compact subset of $\Omega$ such that the $n$-dimensional measure of $F$ is not zero and

$$
\int_{\Omega-F}[\operatorname{dis}(x, F)]^{n-2-\alpha^{\prime}} d x<\infty^{1)}
$$

for some $\alpha^{\prime}$ satisfying

Received February 12, 1982.

1) We denote by $\operatorname{dis}(x, F)$ the distance between $x$ and $F$. 


$$
n c_{1}-2 c_{0}<\alpha^{\prime} c_{0}<(n-1) c_{0}
$$

From now on we write $\hat{x}_{i}=\left(x_{1}, \cdots, x_{i-1}, x_{i+1}, \cdots, x_{n}\right)$ and $x=\left(x_{i}, \hat{x}_{i}\right)$. If we put

$$
G_{\hat{x}_{i}}=F^{c} \cap\left\{\left(x_{1}, \cdots, x_{i-1}, y, x_{i+1}, \cdots, x_{n}\right) \mid y \in R^{1}\right\},
$$

it can be written by

$$
G_{\hat{x}_{i}}=\left(-\infty, a_{0}\right) \cup\left(b_{0}, \infty\right) \cup\left(\bigcup_{\nu=1}^{\infty}\left(a_{\nu}, b_{\nu}\right)\right)
$$

for some $a_{\nu}, b_{\nu}(\nu=0,1,2, \cdots)$, since $G_{\hat{x}_{i}}$ is an open set in $R^{1}$. We assume that there are positive constants $C$ and $\sigma$ with $\sigma<1$ independent of $i$ and $\hat{x}_{i}$ such that

$$
\sum_{\nu=1}^{\infty}\left(b_{\nu}-a_{\nu}\right)^{\sigma} \leqq C
$$

We define

$$
|u|_{s, A}=\sup _{\substack{\ell \leq s \\ x \in A}}\left|\partial_{x_{1}}^{\ell_{1}} \cdots \partial_{x_{n}}^{\ell_{n}} u(x)\right| \quad\left(\ell=\ell_{1}+\cdots+\ell_{n}\right)
$$

for non-negative integers $s$ and subsets $A$ of $\Omega$. As well-known, this norm is extended also for real positive $s$.

Our objective is to prove

TheOREM. Let $\left\{u_{k}\right\}$ be solutions of (1.3) in $\Omega$ and be in $C^{2}(\Omega)$. Let $u_{k} \geqq 0$ in $\Omega$. Assume that $F$ satisfies the above conditions. Then for any $p$ with $1 \leqq p<\infty$ there are positive constants $\gamma(<1), C, d$ and an open subset $G$ of $\Omega$ such that the following holds:

If $\left|u_{k}\right|_{2, \Omega} \leqq K$ and $\left|u_{k}\right|_{1, F} \leqq \varepsilon$ for $\varepsilon, K$ with $0 \leqq \varepsilon \leqq d K$, we have

$$
\left\|u_{k}\right\|_{L^{p}(G)} \leqq C \varepsilon^{\gamma} K^{1-\gamma},
$$

where $r, C, d$ and $G$ depend only on $F, p$ and the coefficients of (1.3).

The proof is given in the final section. In the next section we give an example of compact subsets which satisfies (1.4) and (1.7), but contains neither interior points nor continuous arcs. And for this example we see that the assumption of $\left|u_{k}\right|_{1, F} \leqq \varepsilon$ can be replaced by the weaker one of $\left|u_{k}\right|_{0, F} \leqq \varepsilon$ in our Theorem (see Proposition 2).

The system (1.3) was considered first by K. Akô [1] for more extensive non-linear elliptic operators. He derived the minimum principle for non- 
negative solutions of (1.3) which are named "vector superfunctions" in [1]. When particularly $\varepsilon=0$ in our Theorem, it means that $u_{k}$ vanish identically. A. Ancona [2] has discussed the distribution of zero points for non-negative solutions $u$ of the inequality $\Delta u \leqq \alpha \cdot u$ in the weak sense, where the assumption on $\alpha$ is weaker than ours, that is, $\alpha$ is locally integrable in $\Omega$.

By V. A. Kondrat'ev and S. D. Ėidel'man [6], the Harnack's inequality holds in the wide sense for positive solutions of (1.3). This implies that

$$
\sum_{k=1}^{m} \int_{\Omega^{\prime}} u_{k} d x \leqq C \sum_{k=1}^{m} \int_{\Omega^{\prime \prime}} u_{k} d x
$$

for any two fixed subdomains $\Omega^{\prime}$ and $\Omega^{\prime \prime}$ with $\Omega^{\prime \prime} \subset \Omega^{\prime} \subset \Omega$. Hence our Theorem is effective, when $F$ has no interior point. In [6] more general non-stationary systems were treated. For elliptic systems (1.3) F. Mandras [7] also has shown an inequality of Harnack's type in the ordinary sense, where it needs some hypothesis on the sign of $c_{\ell}^{(k)}(x)$.

Recently, the problem of elliptic variational inequalities with obstacles has been studied in great detail for vector-valued functions by several authors (cf. e.g. [4]), which is closely related to inequalities (1.3). In this connection, we give a remark in the final section (see Remark 4).

In category of complex valued functions, a theorem analogous to ours is obtained for elliptic operators with simple characteristics and two variables (see [3]), where the method is restricted to the case of $n=2$ and the motivation is derived from the ill-posed problems.

We have also

Corollary. Let $\left\{u_{k}\right\}$ and $F$ be such as in our Theorem. Then for any $p$ with $1 \leqq p<\infty$ and for any subdomain $\Omega^{\prime}$ with $\Omega^{\prime} \subset \Omega$, there are positive constants $C, d$ and $\gamma(<1)$ depending only on $F, p, \Omega^{\prime}$ and the coefficients of (1.3) such that the following is valid:

If $\left|u_{k}\right|_{2, \Omega} \leqq K$ and $\left|u_{k}\right|_{1, F} \leqq \varepsilon$ for $\varepsilon, K$ with $0 \leqq \varepsilon \leqq d K$, the inequality

$$
\left\|u_{k}\right\|_{L^{p}\left(\Omega^{\prime}\right)} \leqq C \varepsilon^{r} K^{1-r}
$$

holds.

In fact, combining (1.8) and the inequality of [6], we have

$$
\begin{aligned}
\left\|u_{k}\right\|_{L^{p}\left(\Omega^{\prime}\right)} & \leqq K^{(p-1) / p}\left(\int_{\Omega^{\prime}} u_{k} d x\right)^{1 / p} \\
& \leqq C K^{(p-1) / p} \sum_{j=1}^{m}\left(\int_{G} u_{j} d x\right)^{1 / p}
\end{aligned}
$$




$$
\begin{aligned}
& \leqq C K^{(p-1) / p}\left(C \varepsilon^{r} K^{1-r}\right)^{1 / p} \\
& \leqq C \varepsilon^{\gamma / p} K^{(p-r) / p} .
\end{aligned}
$$

Replacing $\gamma / p$ newly with $\gamma$, we obtain our Corollary.

2. In this section we denote the one-dimensional variable by $x$ or $y$. Let $0<\delta<1$ and let $Q=\{(x, y) ;|x|<a,|y|<b\}$, where $b<a<1$. We calculate first the integral

$$
\iint_{Q}\left[\operatorname{dis}\left((x, y), Q^{c}\right)\right]^{-\delta} d x d y .
$$

If we put $Q^{\prime}=\{(x, y) ; 0<b x / a<y<b\}$, we then have

$$
\begin{aligned}
\iint_{Q^{\prime}} & {\left[\operatorname{dis}\left((x, y), Q^{c}\right)\right]^{-\delta} d x d y } \\
& =\iint_{Q^{\prime}}(b-y)^{-\delta} d x d y \\
& =(1-\delta)^{-1}(2-\delta)^{-1} a b^{1-\delta} .
\end{aligned}
$$

If we put

$$
\begin{aligned}
& Q^{\prime \prime}=\{(x, y) ; 0<y<b x / a<b\} \\
& Q_{1}^{\prime \prime}=Q^{\prime \prime} \cap\{0<x<a-b\}, \\
& Q_{2}^{\prime \prime}=Q^{\prime \prime} \cap\{y>x+b-a\} \cap\{x>a-b\}
\end{aligned}
$$

and

$$
Q_{3}^{\prime \prime}=Q^{\prime \prime} \cap\{y<x+b-a\},
$$

it follows that

$$
\operatorname{dis}\left((x, y), Q^{c}\right)= \begin{cases}b-y & \text { in } Q_{1}^{\prime \prime} \cup Q_{2}^{\prime \prime} \\ a-x & \text { in } Q_{3}^{\prime \prime}\end{cases}
$$

(see Figure 1).

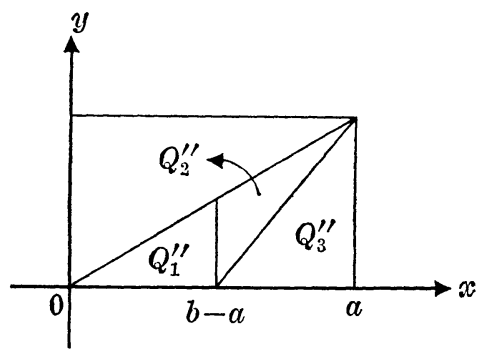

(Figure 1) 
Hence we get

$$
\begin{aligned}
& \iint_{Q_{1}^{\prime \prime}}\left[\operatorname{dis}\left((x, y), Q^{c}\right)\right]^{-\delta} d x d y \\
& \quad=(1-\delta)^{-1}\left[(a-b) b^{1-\delta}+(2-\delta)^{-1}\left(b^{2-\delta}-a^{2-\delta}\right)(b / a)^{1-\delta}\right], \\
& \iint_{Q_{2}^{\prime \prime}}\left[\operatorname{dis}\left((x, y), Q^{c}\right)\right]^{-\delta} d x d y=(1-\delta)^{-1}(2-\delta)^{-1}\left(a^{1-\delta}-b^{1-\delta}\right) a^{\delta-1} b^{2-\delta}
\end{aligned}
$$

and

$$
\iint_{Q_{3}^{\prime \prime}}\left[\operatorname{dis}\left((x, y), Q^{c}\right)\right]^{-\delta} d x d y=(1-\delta)^{-1}(2-\delta)^{-1} b^{2-\delta} .
$$

Combining these equalities we obtain

$$
\iint_{Q}\left[\operatorname{dis}\left((x, y), Q^{c}\right)\right]^{-\delta} d x d y \leqq C(a b)^{1-\delta} .
$$

Secondly we put

$$
A=\{|x|<a / 2,|y|<a / 2\} \cap[\{|x|<b / 2\} \cup\{|y|<b / 2\}]
$$

and

$$
\begin{aligned}
& A_{1}=\{|x|<b / 2,|y|<b / 2\}, \\
& A_{2}=\{|x|<b / 2, b / 2<y<a / 2\}, \\
& A_{3}=\{|x|<b / 2,-a / 2<y<-b / 2\}, \\
& A_{4}=\{-a / 2<x<-b / 2,|y|<b / 2\}, \\
& A_{5}=\{b / 2<x<a / 2,|y|<b / 2\}
\end{aligned}
$$

(see Figure 2).

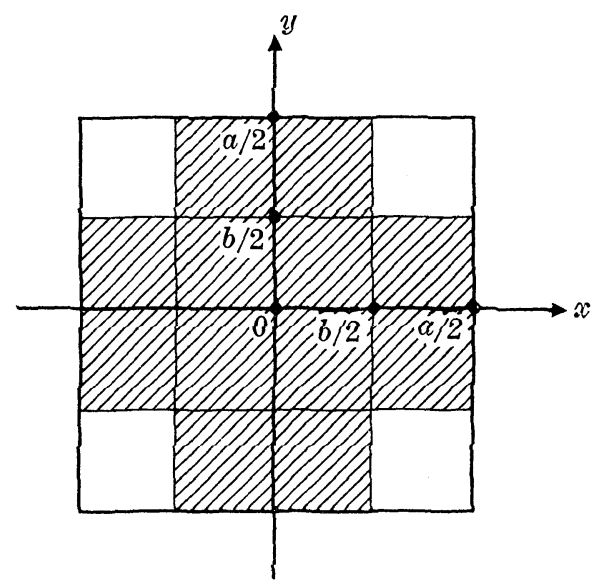

(Figure 2) 
Then $A=\bigcup_{i=1}^{5} A_{i}$ and $\operatorname{dis}\left((x, y), A^{c}\right) \geqq \operatorname{dis}\left((x, y), A_{i}^{c}\right)$. Thus it follows that

$$
\begin{aligned}
\iint_{A}\left[\operatorname{dis}\left((x, y), A^{c}\right)\right]^{-\delta} d x d y \\
\quad \leqq \sum_{i=1}^{5} \iint_{A_{i}}\left[\operatorname{dis}\left((x, y), A_{\imath}^{c}\right)\right]^{-\delta} d x d y .
\end{aligned}
$$

By (2.1) this implies that

$$
\begin{aligned}
\iint_{A}[ & \left.\operatorname{dis}\left((x, y), A^{c}\right)\right]^{-\delta} d x d y \\
& \leqq C\left[b^{2(1-\delta)}+4(b(a-b))^{1-\delta}\right] .
\end{aligned}
$$

Now we give an example of compact subsets $F$ with the assumptions in the previous section.

We consider in a closed interval $[0, c](0<c<1)$. For the time being we take a sequence in such a way that

$$
0<a_{n}<2^{1-n}\left(c-\sum_{j=1}^{\infty} 2^{j-1} a_{j}\right), \quad n=1,2, \cdots .
$$

We eliminate from $[0, c]$ an open interval $O_{1}^{(1)}$ with its length $a_{1}$ and with its center $c / 2$. The remained closed intervals are denoted respectively by $I_{1}^{(1)}$ and $I_{2}^{(1)}$ in turn from the left. Next we eliminate from each $I_{j}^{(1)}$ an open interval $O_{j}^{(2)}$ with its length $a_{2}$ whose center is identical with that of $I_{j}^{(1)}$. Then there are remained four closed intervals which are denoted by $I_{1}^{(2)}, I_{2}^{(2)}, I_{3}^{(2)}$ and $I_{4}^{(2)}$ in turn from the left, respectively (see Figure 3). We repeat inductively this process, that is, $O_{j}^{(n+1)}$ is eliminated from the center of $I_{\jmath}^{(n)}\left(j=1,2, \cdots, 2^{n}\right)$. The lastly remained closed set is written by $E$.

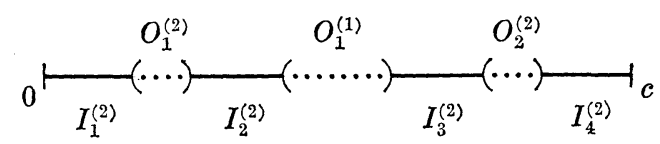

(Figure 3)

Obviously, $|E|=c-\sum_{j=1}^{\infty} 2^{j-1} a_{j}>0^{2)}$. Since

$$
\left|O_{j}^{(n)}\right|=a_{n}, \quad\left|I_{j}^{(n)}\right|=2^{-n}\left(c-\sum_{j=1}^{n} 2^{j-1} a_{j}\right),
$$

we see by (2.3)

2) The notation $|\cdot|$ means the one-dimensional measure. 


$$
\left|I_{j}^{(n)} \cup O_{j^{\prime}}^{(n)}\right||| I_{j}^{(n)} \mid \leqq M,
$$

where $M$ is independent of $n, j$ and $j^{\prime}$.

For the above set $E$ we have

Proposition 1. Suppose that $u \in C^{2}([0, c])$ and $\left|u^{\prime \prime}\right| \leqq K$ in $[0, c]$. If $|u| \leqq \varepsilon(\leqq K)$ on $E$, it follows that

$$
\left|u^{\prime}\right| \leqq C_{0} \sqrt{\varepsilon \bar{K}}
$$

on $E$, where $C_{0}$ is independent of $\varepsilon$ and $K$.

Proof. First we note that this proposition is correct, if $|u| \leqq \varepsilon$ and $u^{\prime} \neq 0$ in $[0, c]$. In fact, we have for some $\xi$

$$
\left|u^{\prime}(\xi)\right|=|(u(c)-u(0)) /(c-0)| \leqq 2 \varepsilon / c
$$

and in virtue of Cauchy's mean value theorem we get

$$
\left|\left(u^{\prime}(x)^{2}-u^{\prime}(\xi)^{2}\right) /(u(x)-u(\xi))\right|=2\left|u^{\prime}\left(\xi^{\prime}\right) u^{\prime \prime}\left(\xi^{\prime}\right) / u^{\prime}\left(\xi^{\prime}\right)\right| \leqq 2 K
$$

for any $x \in(0, c)$. Thus (2.5) is obtained.

Now, by the uniform continuity of $u$ there is a number $n$ such that $|u| \leqq 2 \varepsilon$ on $\bigcup_{j=1}^{2 n} I_{j}^{(n)}$, since $\bigcup_{j=1}^{2 n} I_{j}^{(n)} \searrow E$ as $n \rightarrow \infty$. It is sufficient to prove (2.5) on each $I_{k}^{(n)}$ for such $n$ and for any fixed $k$.

Naturally it may be assumed that $\check{I}_{k}^{(n)} \cap\left\{u^{\prime}(x) \neq 0\right\} \neq \phi$, where $\check{I}_{k}^{(n)}$ is the interior of $I_{k}^{(n)}$. This set can be written as a union of open connected components, that is, $\stackrel{\circ}{i}_{k}^{(n)} \cap\left\{u^{\prime}(x) \neq 0\right\}=\bigcup_{j=1}^{\infty}\left(\gamma_{j}, \delta_{j}\right)$. It suffices to show (2.5) for each $\left(\gamma_{j}, \delta_{j}\right)$. If $u^{\prime}\left(\gamma_{j}\right)=0$, it follows from the Cauchy's mean value theorem that

$$
\left(u^{\prime}(x)^{2}-u^{\prime}\left(\gamma_{j}\right)^{2}\right) /\left(u(x)-u\left(\gamma_{j}\right)\right)=2 u^{\prime \prime}(\xi)
$$

for any $x \in\left(\gamma_{j}, \delta_{j}\right)$ and for some $\xi \in\left(\gamma_{j}, x\right)$. Thus (2.5) holds in $\left(\gamma_{j}, \delta_{j}\right)$. It is similar, if $u^{\prime}\left(\delta_{j}\right)=0$.

When $u^{\prime}\left(\gamma_{j}\right) \neq 0$ and $u^{\prime}\left(\delta_{j}\right) \neq 0$, it is clear that $\check{I}_{k}^{(n)}=\left(\gamma_{j}, \delta_{j}\right)$ and $u^{\prime} \neq 0$ on $I_{k}^{(n)}$. Hence $u^{\prime}>0$ or $u^{\prime}<0$ there. We may assume that $u^{\prime}>0$ on $I_{k}^{(n)}$ without loss of generality. It is immediately seen that some $O_{k^{\prime}}^{(n)}$ is adjacent either on the left or right side of $I_{k}^{(n)}$. For example, let $O_{k^{\prime}}^{(n)}$ be on the right side of $I_{k}^{(n)}$. Let $I_{k}^{(n)}=[\gamma, \delta]$, and let us put

$$
\eta_{0}=\sup \left\{\eta \mid u^{\prime}>0 \text { on }[\gamma, \eta], \gamma<\eta \leqq c\right\} .
$$

Then $\delta<\eta_{0} \leqq c$. It suffices to assume that $u^{\prime}\left(\eta_{0}\right)=0$. In fact, unless it is so, we may treat $\eta_{0}^{\prime}$ in place of $\eta_{0}$ such as 


$$
\eta_{0}^{\prime}=\inf \left\{\eta \mid u^{\prime}>0 \text { on }[\eta, \delta], 0 \leqq \eta<\delta\right\} .
$$

When $\eta_{0} \in \bar{O}_{k^{\prime}}^{(n)}$, we see that

$$
u^{\prime}\left(x_{0}\right)^{2}=\frac{u(\delta)-u(\gamma)}{(\delta-\gamma)^{2}}(u(\delta)-u(\gamma))
$$

for some $x_{0} \in(\gamma, \delta)$, and

$$
u(\delta)-u(\gamma)=(\delta-\gamma) u^{\prime}(\gamma)+\frac{1}{2}(\delta-\gamma)^{2} u^{\prime \prime}\left(x_{1}\right)
$$

for some $x_{1} \in(\gamma, \delta)$. Since $\gamma, \delta \in E$, this implies

$$
u^{\prime}\left(x_{0}\right)^{2} \leqq 4 \varepsilon\left(\left|u^{\prime}(\gamma)\right| /(\delta-\gamma)+\frac{1}{2} K\right) .
$$

On the other hand it follows from (2.4) that

$$
\begin{aligned}
\left|u^{\prime}(\gamma)\right| /(\delta-\gamma) & \leqq M\left|u^{\prime}\left(\eta_{0}\right)-u^{\prime}(\gamma)\right| /\left(\eta_{0}-\gamma\right) \\
& \leqq M K
\end{aligned}
$$

Hence we obtain $u^{\prime}\left(x_{0}\right)^{2} \leqq 2(2 M+1) \varepsilon K$. Applying again Cauchy's mean value theorem, we have

$$
\left|\left(u^{\prime}(x)^{2}-u^{\prime}\left(x_{0}\right)^{2}\right) /\left(u(x)-u\left(x_{0}\right)\right)\right| \leqq 2 K,
$$

for any $x \in[\gamma, \delta]$, that is, $u^{\prime}(x)^{2} \leqq u^{\prime}\left(x_{0}\right)^{2}+2 K\left(|u(x)|+\left|u\left(x_{0}\right)\right|\right)$. Therefore we conclude that on $I_{k}^{(n)}$

$$
u^{\prime}(x)^{2} \leqq 2(2 M+5) \varepsilon K .
$$

This means that (2.5) holds in $I_{k}^{(n)}$.

Next let $\eta_{0} \notin \bar{O}_{k^{\prime}}^{(n)}$. Then we write by $\mu$ the right terminus of $O_{k^{\prime}}^{(n)}$. Naturally $\mu \in E$ and $u^{\prime}>0$ in $[\gamma, \mu]$. Hence we see

$$
-\varepsilon \leqq u(\gamma) \leqq u(x) \leqq u(\mu) \leqq \varepsilon
$$

for $x \in[\gamma, \mu]$, that is, $|u(x)| \leqq \varepsilon$ in $I_{k}^{(n)} \cup O_{k^{\prime}}^{(n)}$. By the way of making $E$ there are $I_{k^{\prime \prime}}^{(n-1)}$ such that $I_{k^{\prime \prime}}^{(n-1)} \supset I_{k}^{(n)}$ and $I_{k^{\prime \prime}}^{(n-1)}=I_{k}^{(n)} \cup O_{k^{\prime}}^{(n)} \cup I_{k+1}^{(n)}$. Since $|u| \leqq 2 \varepsilon$ on $I_{k+1}^{(n)}$, the same inequality holds also on $I_{k^{\prime \prime}}^{(n-1)}$.

If $\eta_{0} \in I_{k+1}^{(n)}$, the Cauchy's mean value theorem implies immediately that $u^{\prime}(x)^{2} \leqq 8 \varepsilon K$ on $I_{k}^{(n)}$, that is, (2.5) holds in $I_{k}^{(n)}$.

If $\eta_{0} \notin I_{k+1}^{(n)}, u^{\prime}>0$ on $I_{k^{\prime \prime}}^{(n-1)}$. Hence replacing $I_{k}^{(n)}$ by $I_{k^{\prime \prime}}^{(n-1)}$, we can repeat the above argument. Doing inductively over and over again, we finally arrive at the case that $u^{\prime}>0$ in $[0, c]$. This is nothing but the beginning of the proof. Thus we complete the proof. 
We set $F=E \times E(\subset \Omega)$ for the above set $E$. From Proposition 1 we have immediately

Proposition 2. Let $u \in C^{2}(\Omega)$, and let $|u|_{2, \Omega} \leqq K$. If $|u|_{0, F} \leqq \varepsilon(\leqq K)$ on $F$, then $|u|_{1, F} \leqq C_{0} \sqrt{\varepsilon \bar{K}}$.

Let $\alpha^{\prime}$ be the constant in (1.5) for $n=2$ and $\sigma$ be any fixed positive number less than 1 . In addition to (2.3) we impose on $E$ the following conditions:

$$
\sum_{j=1}^{\infty} 2^{j-1} a_{j}^{\sigma}<\infty
$$

and

$$
\sum_{j=1}^{\infty} 4^{j} a_{j}^{2\left(1-\alpha^{\prime}\right)}, \quad \sum_{j=1}^{\infty} 4^{j}\left(2^{1-j} a_{j}\right)^{1-\alpha^{\prime}}<\infty .
$$

Then we see that $F$ satisfies (1.4) and (1.7).

In fact, (1.7) is trivial from (2.7). We write $J=[0, c] \times[0, c]$. By virtue of (2.2) we easily see

$$
\begin{aligned}
& \iint_{J-F}[\operatorname{dis}((x, y), F)]^{-\alpha^{\prime}} d x d y \\
& \quad \leqq C\left[\sum_{j=1}^{\infty} 4^{j-1} a_{j}^{2\left(1-\alpha^{\prime}\right)}+\sum_{j=1}^{\infty} 4^{j}\left(2^{1-j}\left(c-\sum_{k=1}^{j} 2^{k-1} a_{k}\right) a_{j}\right)^{1-\alpha^{\prime}}\right] .
\end{aligned}
$$

The sums on the right-hand side is finite from (2.8), which implies immediately (1.4). It is clear that there is actually a sequence which satisfies (2.3), (2.7) and (2.8) simultaneously.

If $F$ is particularly the above set in our Theorem, the assumption $\left|u_{k}\right|_{1, F} \leqq \varepsilon$ is weakened by $\left|u_{k}\right|_{0, F} \leqq \varepsilon$. And (1.8) becomes

$$
\begin{aligned}
\left\|u_{k}\right\|_{L^{p}(G)} & \leqq C\left(C_{0} \sqrt{\varepsilon K}\right)^{\gamma} K^{1-\gamma} \\
& \leqq C^{\prime} \varepsilon^{\gamma / 2} K^{1-\gamma / 2}
\end{aligned}
$$

if $0 \leqq \varepsilon \leqq d^{2} K / C_{0}^{2}$.

3. From now on let $\alpha$ be a fixed number such that

$$
n c_{1}-2 c_{0}<\alpha c_{0}, \quad \alpha<\alpha^{\prime} .
$$

We rewrite (1.1) sinæply by

$$
L=\sum_{i, j} a_{i j} \partial_{x_{i}} \partial_{x_{j}}
$$

where $a_{i j}$ satisfy the assumptions in (1.2). 
Let $r$ be the Euclidean metric of $x$. By (1.2) we have

$$
\begin{aligned}
L r^{-\alpha}= & \alpha(2+\alpha) r^{-4-\alpha} \sum_{i, j} a_{i j} x_{i} x_{j}-\alpha r^{-2-\alpha} \sum_{i} a_{i i} \\
& \geqq \alpha\left(\alpha c_{0}-\left(n c_{1}-2 c_{0}\right)\right) r^{-2-\alpha} .
\end{aligned}
$$

Hence it follows from (3.1) that

$$
L r^{-\alpha} \geqq c_{2} r^{-2-\alpha}
$$

in $\Omega$ for some positive constant $c_{2}$.

We construct the function

$$
\psi_{F}(x)=\int_{F}|x-y|^{-\alpha} d y
$$

for the set $F$ in the first section.

First we see that $\psi_{F}$ is in $C^{1}\left(R^{n}\right)$. In fact, let $f_{\delta}(r)(\delta>0)$ be a $C^{1}$ function such that

$$
\left|\partial_{r} f_{\delta}(r)\right| \leqq 1 \quad \text { in } r \geqq 0
$$

and

$$
\begin{array}{ll}
f_{\delta}(r)=r & \text { for } r \geqq \delta, \\
\delta / 2 \leqq f_{\delta}(r) \leqq \delta & \text { for } r \leqq \delta .
\end{array}
$$

If we set

$$
\psi_{F}^{(\delta)}(x)=\int_{F}\left(f_{\delta}(|x-y|)\right)^{-\alpha} d y
$$

we obtain

$$
\begin{aligned}
& \left|\partial_{x_{i}} \psi_{F}^{(\delta)}(x)-\int_{F} \partial_{x_{i}}\left(|x-y|^{-\alpha}\right) d y\right| \\
& \quad \leqq \alpha\left(\int_{F \cap D_{\delta}}\left|\partial_{r} f_{\delta}\right| f^{-1-\alpha} d y+\int_{F^{\prime} \cap D_{\delta}}|x-y|^{-1-\alpha} d y\right),
\end{aligned}
$$

where $D_{\delta}=\{y ;|x-y| \leqq \delta\}$. By (1.5) and (3.1) the right-hand side tends to zero uniformly as $\delta \rightarrow 0$. Hence $\psi_{F} \in C^{1}\left(R^{n}\right)$.

Next we have

$$
\left|\partial^{2} \psi_{F}(x)\right| \leqq C[\operatorname{dis}(x, F)]^{n-2-\alpha^{\prime}} .
$$

Indeed, since dis $(x, F) \leqq|x-y|$ for $y \in F$, it follows that in $F^{c}$

$$
\begin{aligned}
\left|\partial^{2} \psi_{F}(x)\right| & \leqq C \int_{F}|x-y|^{-2-\alpha} d y \\
& \leqq C[\operatorname{dis}(x, F)]^{n-2-\alpha^{\prime}} \int_{F}|x-y|^{\mid \alpha^{\prime}-\alpha-n} d y .
\end{aligned}
$$


The last integral is uniformly bounded by virtue of $\alpha^{\prime}>\alpha$. Hence (3.3) is valid.

Lemma $1^{3)}$. For any given $\rho>0$, there are positive numbers $c_{3}, c_{4}$ $\left(c_{3}<c_{4}\right)$, a closed subset $F^{\prime}$ of $F$, and subdomains, $\Omega_{1}, \Omega_{2}, \Omega^{\prime}$ of $\Omega$ with $F^{\prime}$ $\subset \Omega_{1} \subset \Omega_{2} \subset \Omega^{\prime} \subset \Omega$ such that

(i) $F^{\prime}$ has the same assumptions as $F$.

(ii) $\psi_{F^{\prime}}(x),\left|\partial \psi_{F^{\prime}}(x)\right| \leqq \rho L \psi_{F^{\prime}}(x)$ in $\Omega^{\prime}-F^{\prime}$.

(iii) $\psi_{F^{\prime}}(x) \geqq c_{4}$ in $\Omega_{1}$ and $\psi_{F^{\prime}}(x) \leqq c_{3}$ in $\Omega^{\prime}-\Omega_{2}$.

Proof. We may assume that the origin is the Lebesgue density point of $F$ without loss of generality.

By (3.2) there are a positive number $\delta$ and a compact subdomain $\Omega^{\prime}$ of $\Omega$ containing the origin such that if $|y| \leqq \delta$ and $x \in \Omega^{\prime}$, then

$$
|x-y|^{-\alpha}, \quad\left|\partial_{x}\left(|x-y|^{-\alpha}\right)\right| \leqq \rho L_{x}\left(|x-y|^{-\alpha}\right) .
$$

Secondly we choose subdomains, $\Omega_{1}, \Omega_{2}$ of $\Omega$ and $a_{1}, a_{2}$ so that $0 \in \Omega_{1} \subset \Omega_{2}$ $\subset \Omega^{\prime}, 0<a_{1}<a_{2}$ and

$$
|x-y| \begin{cases}\leqq a_{1} & \left(x \in \Omega_{1},|y| \leqq \delta\right), \\ \geqq a_{2} & \left(x \notin \Omega_{2},|y| \leqq \delta\right),\end{cases}
$$

where $\delta$ is retaken, if necessary.

Since the origin is the density point of $F$, there is a closed cube $J$ such that $J \subset\{x \mid r<\delta\} \cap \Omega_{1}$ and the $n$-dimensional measure of $F \cap J$ is not zero. If we put $F^{\prime}=F \cap J$, naturally $F^{\prime}$ is closed and satisfies (1.7). In addition, we see

$$
\begin{aligned}
\int_{\Omega-F^{\prime}} & {\left[\operatorname{dis}\left(x, F^{\prime}\right)\right]^{n-2-\alpha^{\prime}} d x } \\
= & \int_{\Omega-F}\left[\operatorname{dis}\left(x, F^{\prime}\right)\right]^{n-2-\alpha^{\prime}} d x+\int_{F-F^{\prime}}\left[\operatorname{dis}\left(x, F^{\prime}\right)\right]^{n-2-\alpha^{\prime}} d x .
\end{aligned}
$$

The first integral of the right-hand side is estimated from the above by

$$
\int_{\Omega-F}[\operatorname{dis}(x, F)]^{n-2-\alpha^{\prime}} d x
$$

and the second integral is estimated by

$$
\int_{F-J}[\operatorname{dis}(x, J)]^{n-2-\alpha^{\prime}} d x \text {. }
$$

Hence $F^{\prime}$ also satisfies (1.4).

3) This appears also in [3] for $n=2$. We repeat its proof in order to make sure. 
The statement (i) has just been shown. In view of (3.4), (ii) is valid. Putting $c_{3}=a_{2}^{-\alpha}$ mes $F^{\prime}$ and $c_{4}=a_{1}^{-\alpha}$ mes $F^{\prime}$, we have (iii). The proof is complete.

4. Let $\sigma$ be as in (1.7) and $c_{5}$ be a fixed positive number such that $\psi_{F^{\prime}}<c_{5}$ in $\Omega^{\prime}$. Then we have

LEMMA 2. Let $v \in C^{2}\left(\Omega^{\prime}\right)$ with compact support in $\Omega^{\prime}$ and $v \geqq 0$ in $\Omega^{\prime}$. If $|v|_{2, \Omega^{\prime}} \leqq K$ and $|v|_{1, F^{\prime}} \leqq \varepsilon(\leqq K)$, it follows that for any $\lambda \geqq \lambda_{0}(>0)$

$$
\begin{aligned}
& \int_{F^{\prime c}} v L^{*}\left(\exp \left(\lambda \psi_{F^{\prime}}\right)\right) d x \\
& \quad \leqq C\left(\varepsilon^{1-\sigma} K^{\sigma} e^{2 \lambda c_{5}}+\int_{F^{\prime \prime}} L v \cdot \exp \left(\lambda \psi_{F^{\prime}}\right) d x\right)^{4)},
\end{aligned}
$$

where $C, \lambda_{0}$ are independent of $\varepsilon$ and $K$.

Proof. For brevity we denote by $\psi$ and $\varphi$ the functions $\psi_{F^{\prime}}$ and $\exp \left(\lambda \psi_{F^{\prime}}\right)$ respectively.

We replace $F$ by $F^{\prime}$ in the definition of $G_{\hat{x}_{i}}$. Let the new $G_{\hat{x}_{i}}$ be rewritten in the same form as (1.6). We note that the points $\left(a_{\nu}, \hat{x}_{i}\right)$ and $\left(b_{\nu}, \hat{x}_{i}\right)$ are all in $F^{\prime}$. For $g \in C^{2}\left(\Omega^{\prime}\right)$ we see that

$$
\begin{aligned}
\int_{a_{\nu}}^{b_{\nu}} v g \partial_{x_{i}} \partial_{x_{j}} \varphi d x_{i}= & {\left[\left(v g \partial_{x_{j}} \varphi\right)\left(y, \hat{x}_{i}\right)\right]_{y=a_{\nu}}^{y=b_{\nu}} } \\
& -\int_{a_{\nu}}^{b_{\nu}} \partial_{x_{i}}(v g) \cdot \partial_{x_{j}} \varphi d x_{i}
\end{aligned}
$$

and

$$
\begin{aligned}
{\left[\left(v g \partial_{x_{j}} \varphi\right)\left(y, \hat{x}_{i}\right)\right]_{y=a_{\nu}}^{y=b_{\nu}}=} & \left(g \partial_{x_{j}} \varphi\right)\left(b_{\nu}, \hat{x}_{i}\right)\left(v\left(b_{\nu}, \hat{x}_{i}\right)-v\left(a_{\nu}, \hat{x}_{i}\right)\right) \\
& +v\left(a_{\nu}, \hat{x}_{i}\right)\left(\partial_{x_{j}} \varphi\right)\left(b_{\nu}, \hat{x}_{i}\right)\left(g\left(b_{\nu}, \hat{x}_{i}\right)-g\left(a_{\nu}, \hat{x}_{i}\right)\right) \\
& +(g v)\left(a_{\nu}, \hat{x}_{i}\right)\left(\left(\partial_{x_{j}} \varphi\right)\left(b_{\nu}, \hat{x}_{i}\right)-\left(\partial_{x_{j}} \varphi\right)\left(a_{\nu}, \hat{x}_{i}\right)\right) .
\end{aligned}
$$

We use the trivial inequality

$$
|X-Y| \leqq C\left(X^{1-\sigma}+Y^{1-\sigma}\right)|X-Y|^{\sigma} \quad(X, Y \geqq 0) .
$$

It follows from the mean value theorem that

$$
\begin{aligned}
\left|v\left(b_{\nu}, \hat{x}_{i}\right)-v\left(a_{\nu}, \hat{x}_{i}\right)\right| \\
\quad \leqq C\left(v\left(a_{\nu}, \hat{x}_{i}\right)^{1-\sigma}+v\left(b_{\nu}, \hat{x}_{i}\right)^{1-\sigma}\right)\left|v\left(b_{\nu}, \hat{x}_{i}\right)-v\left(a_{\nu}, \hat{x}_{i}\right)\right|^{\sigma} \\
\quad \leqq C \varepsilon^{1-\sigma} K^{\sigma}\left(b_{\nu}-a_{\nu}\right)^{\sigma} .
\end{aligned}
$$

Hence, noting that $\left|\partial_{x_{j}} \varphi\right| \leqq C \lambda e^{\lambda c_{5}}$, we obtain

4) $L^{*}$ is the formal adjoint operator of $L$. 


$$
\begin{aligned}
& \left|\left[\left(v g \partial_{x_{j}} \varphi\right)\left(y, \hat{x}_{i}\right)\right]_{y=a_{\nu}}^{y=b_{\nu}}\right| \\
& \qquad C\left(\lambda e^{\lambda c_{5} \varepsilon^{1-\sigma} K^{\sigma}\left(b_{\nu}-\right.} a_{\nu}\right)^{\sigma}+\varepsilon \lambda e^{\lambda c_{5}}\left(b_{\nu}-a_{\nu}\right) \\
& \left.\quad+\varepsilon \int_{a_{\nu}}^{b_{\nu}}\left|\left(\partial_{x_{i}} \partial_{x_{j}} \varphi\right)\left(x_{i}, \hat{x}_{i}\right)\right| d x_{i}\right) .
\end{aligned}
$$

Since $\left|\partial^{2} \varphi\right| \leqq \lambda\left(\left|\partial^{2} \psi\right|+\lambda|\nabla \psi|^{2}\right) \varphi$, this implies that

$$
\begin{aligned}
& \left|\left[\left(v g \partial_{x_{j}} \varphi\right)\left(y, \hat{x}_{i}\right)\right]_{y=a_{\nu}}^{y=b_{\nu}}\right| \\
& \quad \leqq C e^{2 \lambda c_{5}}\left(\varepsilon^{1-\sigma} K^{\sigma}\left(b_{\nu}-a_{\nu}\right)^{\sigma}+\varepsilon \int_{a_{\nu}}^{b_{\nu}}\left|\left(\partial^{2} \psi\right)\left(x_{i}, \hat{x}_{i}\right)\right| d x_{i}\right) .
\end{aligned}
$$

We integrate the both sides of (4.1) with $x_{j}$ and repeat the same calculus as there. Then by virtue of (1.4), (1.7) and (3.3) it follows immediately that

$$
\left|\int_{F^{\prime c}} v g \partial_{x_{i}} \partial_{x_{j}} \varphi d x-\int_{F^{\prime} c} \varphi \partial_{x_{j}} \partial_{x_{i}}(g v) d x\right| \leqq C \varepsilon^{1-\sigma} K^{\sigma} e^{2 \lambda c_{5}}
$$

We verify more easily

$$
\left|\int_{F^{\prime c}} v g \partial_{x_{i}} \varphi d x-\int_{F^{\prime c}} \varphi \partial_{x_{i}}(v g) d x\right| \leqq C \varepsilon^{1-\sigma} K^{\sigma} e^{2 \lambda c_{5}} .
$$

Therefore we obtain

$$
\left|\int_{F^{\prime c}} v L^{*} \varphi d x-\int_{F^{\prime c}} \varphi L v d x\right| \leqq C \varepsilon^{1-\sigma} K^{\sigma} e^{2 \lambda c_{5}} .
$$

This completes the proof.

5. We prove our Theorem.

Let $\theta(x)$ be a function in $C^{2}\left(\Omega^{\prime}\right)$ with compact support in $\Omega^{\prime}$ such that $\theta \geqq 0$ in $\Omega^{\prime}$ and $\theta=1$ in $\Omega_{2}$. We set $v_{k}=\theta u_{k}$. Then (1.3) is rewritten by

$$
L_{k} v_{k} \leqq \sum_{i, \ell} b_{i \ell}^{(k)} \partial_{x_{i}} v_{\ell}+\sum_{\ell} c_{\ell}^{(k)} v_{\ell}+R_{k}
$$

where

$$
R_{k}=u_{k} L_{k} \theta+2 \sum_{i, j} a_{i j}^{(k)} \partial_{x_{i}} \theta \cdot \partial_{x_{j}} u_{k}-\sum_{i, \ell} b_{i \ell}^{(k)} \partial_{x_{i}} \theta \cdot u_{\imath} .
$$

By virtue of Lemma 2 it follows that for $\lambda \geqq \lambda_{0}$

$$
\sum_{k=1}^{m} \int_{F^{\prime} c} \imath_{h} L_{k}^{*} \varphi d x \leqq C\left(\varepsilon^{1-\sigma} K^{\sigma} e^{2 \lambda c_{5}}+\sum_{k=1}^{m} \int_{F^{\prime c}} \varphi L_{k} v_{k} d x\right)
$$


Naturally $L_{k}^{*}$ is written:

$$
L_{k}^{*}=L_{k}+\sum_{i} b_{i}^{(k)}(x) \partial_{x_{i}}+c^{(k)}(x) .
$$

Thus we see

$$
L_{k}^{*} \varphi=\left[\lambda^{2} \sum_{i, j} a_{i j}^{(k)} \partial_{x_{i}} \psi \cdot \partial_{x_{j}} \psi+\lambda\left(\sum_{i, j} a_{i j}^{(k)} \partial_{x_{i}} \partial_{x_{j}} \psi+\sum_{i} b_{i}^{(k)} \partial_{x_{i}} \psi\right)+c^{(k)}\right] \varphi .
$$

By (3.2) and ellipticity of $L_{k}$ there are a positive constant $c_{6}$ and $\lambda_{1}\left(\geqq \lambda_{0}\right)$ such that for $\lambda \geqq \lambda_{1}$

$$
L_{k}^{*} \varphi \geqq c_{6} \lambda \varphi\left(\int_{F^{\prime}}|x-y|^{-2-\alpha} d y+\lambda|\nabla \psi|^{2}\right)
$$

in $\Omega^{\prime}-F^{\prime}$.

On the other hand it follows from (4.2) that

$$
\left|\int_{F^{\prime c}} \varphi b_{i \ell}^{(k)} \partial_{x_{i}} v_{\ell} d x\right| \leqq C\left(\varepsilon^{1-\sigma} K^{\sigma} e^{2 \lambda c_{5}}+\int_{F^{\prime c}}(1+\lambda|\partial \psi|) v_{\ell} \varphi d x\right) .
$$

Combining the above inequalities, we obtain for $\lambda \geqq \lambda_{1}$

$$
\int_{F^{\prime \prime}} \varphi\left(\sum_{k} v_{k}\right) d x \leqq C\left(\varepsilon^{1-\sigma} K^{\sigma} e^{2 \lambda c_{5}}+\int_{F^{\prime \prime}} \varphi\left(\sum_{k}\left|R_{k}\right|\right) d x\right),
$$

where it is seen that the integral on the left-hand side can be replaced by

$$
\int \varphi\left(\sum_{k} v_{k}\right) d x \text { since } \int_{F^{\prime}} \varphi\left(\sum_{k} v_{k}\right) d x \leqq C \varepsilon e^{\lambda c_{5}} .
$$

We note that $R_{k}=0$ in $\Omega_{2}$. Reducing the integral domain of the lefthand side, we have by Lemma 1

$$
e^{\lambda c_{1}} \int_{\Omega_{1}}\left(\sum_{k} u_{k}\right) d x \leqq C\left(\varepsilon^{1-\sigma} K^{\sigma} e^{2 \lambda c_{5}}+K e^{\lambda c_{3}}\right) \quad\left(\lambda \geqq \lambda_{1}\right) .
$$

That is,

$$
\int_{\Omega_{1}}\left(\sum_{k} u_{k}\right) d x \leqq C\left(\varepsilon^{1-\sigma} K^{\sigma} e^{\lambda\left(2 c_{5}-c_{4}\right)}+K e^{\lambda\left(c_{3}-c_{4}\right)}\right) .
$$

Now we use the idea of F. John [5] (see p. 559) with respect to the ill-posed Cauchy problem. Setting $h=(1-\sigma)\left(2 c_{5}-c_{3}\right)^{-1}$ and $\lambda=\log (K / \varepsilon)^{h}$, we see

$$
\int_{\Omega_{1}}\left(\sum_{k} u_{k}\right) d x \leqq C \varepsilon^{\beta} K^{1-\beta},
$$

where $\beta=h\left(c_{4}-c_{3}\right)$. Hence we obtain 


$$
\begin{aligned}
\left(\int_{\Omega_{1}} u_{k}^{p} d x\right)^{1 / p} & \leqq\left(K^{p-1} \int_{\Omega_{1}} u_{k} d x\right)^{1 / p} \\
& \leqq C \varepsilon^{\beta / p} K^{1-(\beta / p)} .
\end{aligned}
$$

If we choose $G=\Omega_{1}, \gamma=\beta / p$ and $d=\exp \left(-\lambda_{1} / h\right)$, the proof of our Theorem is complete.

Remark 1. From the above proof we immediately see that the assumption on $c_{\ell}^{(k)}$ is weakened as follows:

$$
c_{\ell}^{(k)}(x)=O\left(\int_{F^{\prime}}|x-y|^{-2-\alpha} d y\right) \quad(\operatorname{dis}(x, F) \rightarrow 0) .
$$

Remark 2. Let $\delta$ be any fixed positive number less than 1 . In our Theorem we easily verify that the assumption $|u|_{2, \Omega} \leqq K$ is weakened to $|u|_{1+\delta, \Omega} \leqq K$, if the real number $\delta$ in (1.7) is restricted to $0<\sigma<\delta$.

Remark 3. Instead of (1.3) we consider the solutions $\left\{u_{k}\right\}$ of the inhomogeneous inequalities

$$
L_{k} u_{k} \leqq \sum_{\ell=1}^{m} \sum_{i=1}^{n} b_{i \ell}^{(k)}(x) \partial_{x_{i}} u_{\ell}+\sum_{\ell=1}^{m} c_{\ell}^{(k)}(x) u_{\ell}+f_{k}, \quad k=1,2, \cdots, m,
$$

where $f_{k} \in C^{0}(\Omega)$. Then (5.2) is replaced with

$$
e^{\lambda c_{4}} \int_{\Omega_{1}}\left(\sum_{k} u_{k}\right) d x \leqq C\left(\varepsilon^{1-\sigma} K^{\sigma} e^{2 \lambda c_{5}}+K e^{\lambda c_{3}}+e^{\lambda c_{5}} \int_{\Omega^{\prime}}\left(\sum_{k}\left|f_{k}\right|\right) d x\right),
$$

which implies that for $\lambda \geqq \lambda_{1}$ we have

$$
\int_{\Omega_{1}}\left(\sum_{k} u_{k}\right) d x \leqq C\left(\varepsilon^{\beta} K^{1-\beta}+(K / \varepsilon)^{h\left(c_{5}-c_{4}\right)} \int_{\Omega^{\prime}}\left(\sum_{k}\left|f_{k}\right|\right) d x\right) .
$$

Remark 4. Let $\left\{u_{k}\right\}$ be in $C^{1+\delta}(\Omega)(0<\delta<1)$ and solutions of (1.3) in the weak sense, that is,

$$
\int u_{k} L_{k}^{*} \phi d x \leqq \sum_{i, \ell} b_{i \ell}^{(k)} \partial_{x_{i}} u_{\ell} \cdot \phi d x+\sum_{\ell} \int c_{\ell}^{(k)} u_{\ell} \phi d x
$$

for any $\phi \in C_{0}^{2}(\Omega)$ with $\phi \geqq 0$ in $\Omega$. We assume (1.7) for $\sigma$ with $0<\sigma<\delta$ and that $L_{k}$ has constant coefficients independent of $k$, which means naturally that $L^{*}=L\left(=L_{k}\right)$. Moreover let $c_{\varepsilon}^{(k)}$ be in $C(\Omega)$.

We construct the well-known regular approximation of $u_{k}$ such that

$$
u_{k}^{(\rho)}(x)=\int u_{k}(y) j_{\rho}(x-y) d y
$$

for sufficiently small $\rho>0$. By (5.3) we have 


$$
\begin{aligned}
L u_{k}^{(\rho)}(x) & =\int u_{k}(y) L^{*} j_{\rho}(x-y) d y \\
& \leqq \sum_{i, \ell} \int b_{i \ell}^{(k)}(y) \partial_{y_{i}} u_{\ell}(y) \cdot j_{\rho}(x-y) d y+\sum_{\ell} \int c_{\ell}^{(k)}(y) u_{\ell}(y) j_{\rho}(x-y) d y \\
& =\sum_{i, \ell} b_{i \ell}^{(k)} \partial_{x_{i}} u_{k}^{(\rho)}+\sum_{\ell} c_{\ell}^{(k)} u_{\ell}^{(\rho)}+f_{k}^{(\rho)} .
\end{aligned}
$$

Here $f_{k}^{(\rho)}$ is of the form:

$$
\begin{aligned}
f_{k}^{(\rho)}(x)= & \sum_{i, \ell} \int\left(b_{i \ell}^{(k)}(y)-b_{i \ell}^{(k)}(x)\right) \partial_{y_{i}} u_{\ell}(y) \cdot j_{\rho}(x-y) d y \\
& +\sum_{\ell} \int\left(c_{\ell}^{(k)}(y)-c_{\ell}^{(k)}(x)\right) u_{\ell}(y) j_{\rho}(x-y) d y
\end{aligned}
$$

Let $\Omega^{\prime \prime}$ be a subdomain such that $\Omega^{\prime} \subset \Omega^{\prime \prime} \subset \Omega$. Since the support of $j_{\rho}(x)$ is in $\{|x|<\rho\}$, it follows that $\left|f_{k}^{(\rho)}\right|_{0, \Omega^{\prime}} \leqq C(\rho)\left(\sum_{\ell}\left|u_{\ell}\right|_{1, \Omega^{\prime \prime}}\right)$, where $C(\rho) \rightarrow 0$ as $\rho \rightarrow 0$.

Let $\varepsilon$ and $K$ be positive numbers in our Theorem. Suppose that $\left|u_{k}\right|_{1, F} \leqq \varepsilon$ and $\left|u_{k}\right|_{1+\delta, \Omega} \leqq K$. Noting that $\left|u_{k}^{(\rho)}-u_{k}\right|_{1, \Omega^{\prime \prime}} \rightarrow 0 \quad(\rho \rightarrow 0)$, we have in view of Remarks 2 and 3

$$
\int_{\Omega_{1}}\left(\sum_{k} u_{k}\right) d x \leqq C \varepsilon^{\beta^{\prime}} K^{1-\beta^{\prime}} \quad\left(0<\beta^{\prime}<1\right) .
$$

Hence the conclusion in our Theorem holds also for this case.

It is known that vector-valued solutions of variational inequalities satisfy (5.3) for some closed convex sets of vector functions. Recently, S. Hildebrandt and K. O. Widman [4] have studied the Hölder continuity for first derivatives of these solutions, where quasi-linear elliptic systems have been treated.

\section{REFERENCES}

[1] K. Akô, Semi-decomposable systems and vector subfunctions, Funkcial. Ekvac., 8 (1966), 91-97.

[2] A. Ancona, Une propriété d'invariance des ensembles absorbants par perturbation d'un opérateur elliptique, Comm. Partial Differential Equations, 4 (1979), 321-337.

[3] K. Hayasida, H. Tachi, On the three circles theorem for solutions of elliptic equations, to appear in Funkcial. Ekvac.

[4] S. Hildebrandt, K. O. Widman, Variational inequalities for vector-valued functions, J. Reine Angew. Math., 309 (1979), 191-220.

[5] F. John, Continuous dependence on data for solutions of partial differential equations with a prescribed bound, Comm. Pure Appl. Math., 8 (1960), 551-585.

[6] V. A. Kondrat'ev, S. D. Èidel'man, Positive solutions of linear partial differential equations, Trans. Moscow Math. Soc., 31 (1974), 81-148. 
[ 7 ] F. Mandras, Disegualianza di Harnack per sistemi ellitici debolmente accoppiati, Boll. Un. Mat. Ital., A (5), 14 (1977), 313-321.

Kazuya HAYASIDA:

Department of Mathematics

Faculty of Science

Kanazawa University

Kanazawa, 920 Japan

Yoshiaki IKEDA:

Department of Mathematics

Aichi University of Education

Kariya, 448 Japan 